\title{
Are all maximally entangled states pure?
}

\author{
D. Cavalcanti* and F. G. S. L. Brandão ${ }^{\dagger}$ \\ Departamento de Física, Caixa Postal 702, Universidade Federal de Minas Gerais, 30123-970 Belo Horizonte, Minas Gerais, Brazil \\ M. O. Terra Cunha \\ Departamento de Matemática, Caixa Postal 702, Universidade Federal de Minas Gerais, 30123-970 Belo Horizonte, \\ Minas Gerais, Brazil \\ (Received 16 May 2005; revised manuscript received 25 July 2005; published 28 October 2005)
}

\begin{abstract}
We study if all maximally entangled states are pure through several entanglement monotones. In the bipartite case, we find that the same conditions which lead to the uniqueness of the entropy of entanglement as a measure of entanglement exclude the existence of maximally mixed entangled states. In the multipartite scenario, our conclusions allow us to generalize the idea of the monogamy of entanglement: we establish the polygamy of entanglement, expressing that if a general state is maximally entangled with respect to some kind of multipartite entanglement, then it is necessarily factorized of any other system.
\end{abstract}

DOI: 10.1103/PhysRevA.72.040303

PACS number(s): 03.67.Mn, 03.65.Ud

One of the most striking differences between classical and quantum correlations is the restricted capability of quantum states to share entanglement. This so-called monogamy of entanglement has been increasingly studied in the past years [1], and is related to the security of quantum cryptographic protocols based on entanglement (it limits the amount of correlations that an eavesdropper can have with the honest parties).

The discussion about the monogamy of entanglement usually begins with the apparent straightforward fact that maximally entangled states are pure. This means that when two systems are as much entangled with each other as it is possible, they cannot be entangled and even classically correlated with anyone else. In this paper we analyze the trustiness of this "common sense" under the view of several entanglement measures. On one hand, it is shown that it is not true for general entanglement monotones, failing at least in the best separable approximation measure [2] and in the indicator measure [3]. On the other hand, we prove that for the majority of entanglement quantifiers maximally entangled states are indeed pure. In particular, we consider the quantifiers related to entanglement witnesses and, especially, the generalized robustness of entanglement. With the help of the witnessed entanglement [4], we introduce the idea of polygamy of entanglement, which states that if a multipartite state is maximally entangled with respect to a given kind of multipartite entanglement, then it must be pure.

In order to avoid future confusion it is important to stress

\footnotetext{
*Electronic address: dcs@ fisica.ufmg.br

${ }^{\dagger}$ Electronic address: fgslb@ufmg.br

*Electronic address: tcunha@mat.ufmg.br

${ }^{1}$ Note that we use the term polygamy in the sense of a marriage among multiple partners. This word has appeared before, in Ref. [5], in the context of symmetric multipartite Gaussian states, as opposed to monogamy, in the sense that states which maximize a certain pair-entanglement quantifier can also maximize the multipartite version of it.
}

that the idea of mixed maximally entangled states (MMES) discussed here is, although related, different from the idea of maximally entangled mixed states (MEMS) presented in Refs. [6] (this is the reason for the exchange of words). In their articles the authors address the following question: what is the highest value of entanglement that states with a given purity (mixing) can present? In our work we study if, given the maximum value of entanglement, there is some mixed state that reaches it.

Before proceeding to show the main result of this communication, we present two simple results, albeit important, valid for every convex quantifier.

Theorem 1. According to all convex entanglement quantifiers there is at least one maximally entangled pure state.

Proof. Given a density operator $\rho=\Sigma_{i} p_{i}\left|\psi_{i}\right\rangle\left\langle\psi_{i}\right|$ and a convex quantity $E$, it holds $E(\rho) \leqslant \Sigma_{i} p_{i} E\left(\left|\psi_{i}\right\rangle\left\langle\psi_{i}\right|\right)$, for every ensemble decomposition $\left\{p_{i}, \psi_{i}\right\}$ of $\rho$. Thus, we see that there must be a $\left|\psi_{i}\right\rangle$ such that $E\left(\left|\psi_{i}\right\rangle\left\langle\psi_{i}\right|\right) \geqslant E(\rho)$.

Moreover, from the convex condition it is easily seen that for a mixed state to be maximally entangled (with respect to $E)$, there must be an ensemble description with all $\left|\psi_{i}\right\rangle$ maximally entangled.

Theorem 2. If $\rho$ is a mixed maximally entangled state with respect to the convex measure $E$, then all states in the subspace spanned by the eigenvectors of $\rho$ are maximally entangled.

Proof. According to the unitary freedom in the ensemble for the density matrices theorem [7], the sets $\left\{p_{i},\left|\psi_{i}\right\rangle\right\}$ and $\left\{q_{j},\left|\phi_{j}\right\rangle\right\}$ generate $\rho$, i.e.,

$$
\rho=\sum_{i} p_{i}\left|\psi_{i}\right\rangle\left\langle\psi_{i}\left|=\sum_{j} q_{j}\right| \phi_{j}\right\rangle\left\langle\phi_{j}\right|,
$$

if and only if $\sqrt{p_{i}}\left|\psi_{i}\right\rangle=\sum_{j} u_{i j} \sqrt{q_{j}}\left|\phi_{j}\right\rangle$, with $\left\{\left|\psi_{i}\right\rangle\right\}$ and $\left\{\left|\phi_{j}\right\rangle\right\}$ being normalized vectors, $u_{i j}$ is a complex unitary matrix, and one can "pad" whichever set of vectors $\left\{\sqrt{p_{i}}\left|\psi_{i}\right\rangle\right\}$ or $\left\{\sqrt{q_{j}}\left|\phi_{j}\right\rangle\right\}$ is smaller with additional null vectors so that the two sets have the same number of elements. 
Since each pure state term in any convex decomposition of $\rho$ must be maximally entangled, we find that the state $\left|\psi_{i}\right\rangle=\Sigma_{j} c_{i j}\left|\phi_{j}\right\rangle$, with coefficients $c_{i j}=u_{i j} \sqrt{q_{j} / p_{i}}$, must be maximally entangled as well. The result follows letting $\left\{\left|\phi_{j}\right\rangle\right\}$ be the eigenvectors of $\rho$ and noting that for a fixed $i$, the vector $c_{i j}$ can have arbitrary elements belonging to the hypersphere $\sum_{j} c_{i j}^{2}=1$.

It is possible to extend entanglement measures defined for pure states to the whole state space with the convex-roof construction. Given the quantity $E$, its convex roof is

$$
E(\rho)=\min _{\left\{p_{i}, \psi_{i}\right\}} p_{i} E\left(\psi_{i}\right) .
$$

It can be shown that $E(\rho)$ is an entanglement monotone whenever $E(\psi)$ is. From Eq. (2) we see that, for convex-roofbased measures, theorem 2 gives necessary and sufficient conditions for the existence of mixed maximally entangled states.

We believe the existence of a $n$-dimensional subspace, with $n \geqslant 2$, formed only by maximally entangled states is a very demanding condition, so that for general convex entanglement measures the maximally entangled states are pure. One might then conjecture that this is true for all entanglement monotones [8]. However, for the indicator measure [3] defined as 1 for entangled states and 0 for separable states, which is obviously an entanglement monotone, every entangled state is maximally entangled. Furthermore, using the result of Ref. [9] which, for every $k$-partite Hilbert space $H$ there exists an entangled subspace of dimension $d_{1} \times d_{2}$ $\times \ldots \times d_{k}-\left(d_{1}+d_{2}+\cdots+d_{k}\right)+k-1$, we find that also for the convex-roof indicator measure maximally entangled states can be mixed.

Theorem 3. According to every bipartite entanglement measure such that all its maximally entangled pure states have the maximum Schmidt rank possible, ${ }^{2}$ there do not exist maximally entangled mixed states.

Proof. By theorem 2, it must exist a subspace of maximally entangled pure states. By hypothesis, they all have maximum Schmidt rank. Take two of them,

$$
|\psi\rangle=\sum_{i j} c_{i j}|i j\rangle, \quad|\phi\rangle=\sum_{i j} d_{i j}|i j\rangle .
$$

If we look at $c_{i j}$ and $d_{i j}$ as coefficients of square matrices $C$ and $D$, the maximum Schmidt rank is equivalent to invertibility of the matrix. However, it always exists $\alpha, \beta \in \mathrm{C}$ such that $\alpha C+\beta D$ is not invertible (take $-\alpha / \beta$ as an eigenvalue of $C^{-1} D$ ), and $\alpha|\psi\rangle+\beta|\phi\rangle$ does not have maximum Schmidt rank.

This theorem applies to a number of important and wellstudied entanglement measures. Consider first the entanglement of formation [10] and the relative entropy of entanglement [3]. They are both convex and equal to the entropy of entanglement $\left(E_{E}\right)$ in pure states. As all maximally entangled states of $E_{E}$ are singlets (which have maximum Schmidt rank), it follows from theorem 3 that neither of them allows

\footnotetext{
${ }^{2}$ The Schmidt rank of a bipartite pure state is the number of nonnull Schmidt coefficients in its Schmidt decomposition.
}

MMES. The same argument is valid for the negativity [11] and the concurrence [12]. We can go even further and establish the following result:

Theorem 4. For all asymptotic continuous and partially additive entanglement monotones, all maximally entangled states are pure.

Proof. From the uniqueness theorem for entanglement measures [13], we have that every entanglement measure $E$ fulfilling the conditions of the theorem obey $E_{D} \leqslant E \leqslant E_{F}$, where $E_{D}$ and $E_{F}$ are the distillable entanglement and the entanglement of formation, respectively. Hence the result follows straightforwardly from the fact that $E_{F}$ does not have MMES and $E_{D}=E_{F}$ for pure states.

The situation is much more subtle when we are dealing with multipartite entanglement. In this case we have to specify which kind of entanglement we are talking about [14], because we could be interested in studying the entanglement among different partitions of the whole system. Furthermore, in the multipartite context, other relevant questions arise in order to classify entangled states, as there are different classes of inequivalent states under SLOCC [15]. Thus, answering if a state is more entangled than another will depend on what criterion one is adopting.

Consider an $m$-partite state with Hilbert space $H=$ $\otimes_{i=1}^{m} H_{i}$. We call $P_{k}^{m}=\left\{A_{j}\right\}_{j=1}^{N}$ a $k$-partition of $\{1,2, \ldots, m\}$ if (i) $A_{j} \subset\{1,2, \ldots, m\}$; (ii) $A_{i} \cap A_{j}=\varnothing, \forall i \neq j$; (iii) $\cup_{i} A_{i}=\{1,2, \ldots, m\}$; (iv) $\sharp\left\{A_{j}\right\} \leqslant k$. The number $k$ is called the length of the partition $P_{k}^{m}$. The set of all $k$-partitions of $\{1,2, \ldots, m\}$ will be denoted by $\mathcal{P}_{k}^{m}$. With this concept, one can define factorizability and separability subjected to a partition, and also subjected only to the length of the partitions.

Definition 1. We say that a state $\rho$ is $P_{k}^{m}$-factorizable if, for a fixed $P_{k}^{m}$, it can be written as $\rho=\rho_{A_{1}} \otimes \ldots \otimes \rho_{A_{n}}$, where $\rho_{A_{j}}$ is a density operator on $H_{A_{j}}=\otimes_{i \in A_{j}} H_{i}$. $\mathrm{A}$ state is $P_{k}^{m}$-separable if it can be written as a convex combination of $P_{k}^{m}$-factorizable states. Finally, we call $k$-separable a state $\rho$ which can be written as a convex combination of $P_{k}^{m}$-factorizable states, where $P_{k}^{m}$ may vary for each pure state.

Let us denote $S_{k}(H)$ the set of $k$-separable states on $H$. Clearly they form a chain $S_{m}(H) \subset S_{m-1}(H) \subset \cdots \subset S_{2}(H) \subset S_{1}(H)=D(H)$, where $D(H)$ denotes the set of density operators on $H$. As each of these sets is closed and convex, there exists a Hermitian operator $W$ such that $\operatorname{Tr}(W \rho)<0$, and $\operatorname{Tr}(W \sigma) \geqslant 0 \forall \sigma \in S_{k}(H)$ [16]. One calls such $W$ a $k$-entanglement witness.

Although several entanglement monotones applicable to multipartite states are known $[1,3,4,17,18]$, only two approaches, up to now, can be applied to the quantification of the different kinds of multipartite entanglement discussed above: ${ }^{3}$ the relative entropy of entanglement [3] and its related measures $[3,17]$, and the witnessed entanglement [4]. The first, with great importance in the bipartite scenario, is based on the minimization of some distance between the

\footnotetext{
${ }^{3}$ The other measures are either based on bipartite entanglement concepts, such as the localizable entanglement, or can only distinguish entangled from fully separable states.
} 
state under question and the sets $S_{k}(H)$. The second, recently introduced in Ref. [4], includes several well-studied bipartite and multipartite entanglement measures and quantifies entanglement based on the concept of optimal entanglement witnesses. In this paper, due to some particular properties, such as the linearity of the objective function, we will consider the witnessed entanglement.

Definition 2. For an $m$-partite state $\rho \in D(H)$, its witnessed $k$-partite entanglement is given by

$$
E_{W}^{k}(\rho)=\max \left\{0,-\min _{W \in \mathcal{M}} \operatorname{Tr}(W \rho)\right\},
$$

where $\mathcal{M}=\mathcal{W}_{k} \cap \mathcal{C}, \mathcal{W}_{k}$ is the set of $k$-entanglement witnesses and $\mathcal{C}$ is some set such that $\mathcal{M}$ is compact.

Having this definition in mind we can see what restrictions are imposed by the existence of a MME state $\rho$ on its optimal entanglement witness $W$.

Let $\rho=\Sigma_{j} q_{j}\left|\phi_{j}\right\rangle\left\langle\phi_{j}\right|$ be the spectral decomposition of $\rho$, and $\left\{p_{i},\left|\psi_{i}\right\rangle\right\}$ another ensemble describing it. Then, $\left|\psi_{i}\right\rangle=\sum_{j} c_{i j}\left|\phi_{j}\right\rangle$, with coefficients $c_{i j}=u_{i j} \sqrt{q_{j} / p_{i}}$. In the case where $\rho$ is maximally entangled with entanglement $E, W$ must be optimal for every $\left|\psi_{i}\right\rangle$ and $\left|\phi_{j}\right\rangle$, which allows us to write for one specific element $\left|\psi_{k}\right\rangle$,

$$
\begin{aligned}
-E & =\operatorname{Tr}\left(W\left|\psi_{k}\right\rangle\left\langle\psi_{k}\right|\right) \\
& =\sum_{i}\left|c_{k i}\right|^{2}\left\langle\phi_{i}|W| \phi_{i}\right\rangle+\sum_{i \neq j} c_{k i}^{*} c_{k j}\left\langle\phi_{i}|W| \phi_{j}\right\rangle \\
& =-E+\sum_{i \neq j} c_{k i}^{*} c_{k j}\left\langle\phi_{i}|W| \phi_{j}\right\rangle,
\end{aligned}
$$

which implies

$$
\sum_{i \neq j} c_{k i}^{*} c_{k j}\left\langle\phi_{i}|W| \phi_{j}\right\rangle=0
$$

As this equality must be true for every ensemble describing $\rho,\left\langle\phi_{i}|W| \phi_{j}\right\rangle=0$ and $W$ is proportional to the identity matrix in the support of $\rho$, with $-E$ as the proportionality constant. Being that $E$ the highest value of entanglement and, therefore, the modulus of the lowest eigenvalue possible among all entanglement witnesses, each eigenvector $\left|\phi_{j}\right\rangle$ of $\rho$ is an eigenvector of $W$ too. Thus $W$ can be written as

$$
W=\underbrace{(-E) I}_{\operatorname{Supp}(\rho)} \oplus \underbrace{D}_{\operatorname{Supp}^{\perp}(\rho)}
$$

where $D$ is some matrix such that the constraints imposed by $\mathcal{C}$ are satisfied. Here again this demanding condition is not sufficient to rule out the existence of mixed maximally entangled states. As a counterexample, consider the best separable approximation measure (BSA) [2], $B_{\text {app }}^{k}(\rho)=1-\lambda$, where $\lambda$ is the optimal value of the following optimization problem:

$$
\max \lambda, \quad \text { s.t. } \rho=\lambda \sigma+(1-\lambda) \pi,
$$

with $\sigma \in S_{k}(H), \pi \in D(H)$, and $\lambda \in[0,1]$. It can be written alternatively as Eq. (4) with $\mathcal{C}=\{W \mid W \geqslant-I\}$ [4]. For the following family of mixed states

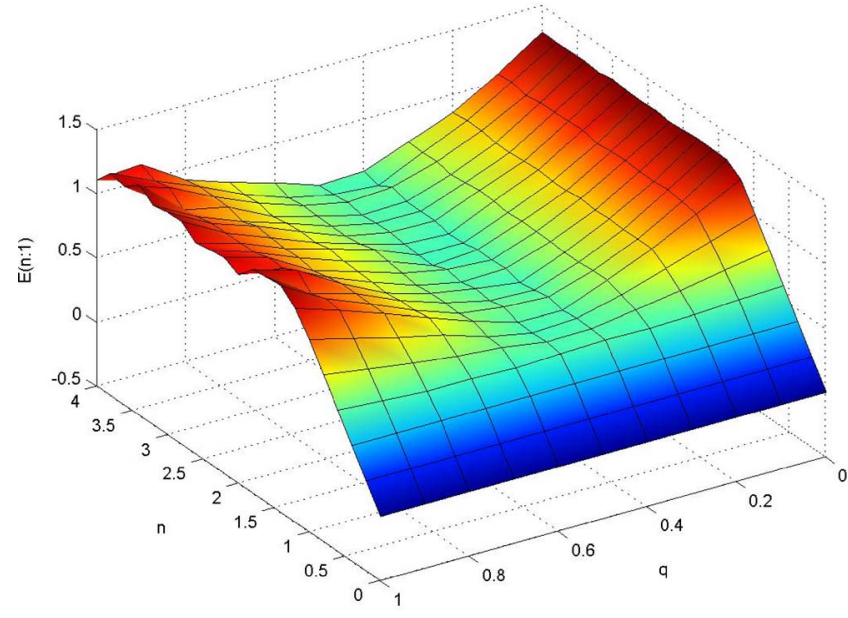

FIG. 1. (Color online) $E_{n: 1}^{1}\left(\rho_{q}\right)$ for $0 \leqslant n \leqslant 4$ and $0 \leqslant q \leqslant 1$. When $n \ll 1, E_{n: 1}^{1}(\rho)=n B_{\text {app }}(\rho)$ and MME states are possible. In the other limit $E_{n: 1}^{1}(\rho)=R^{1}(\rho)$ [4], and all maximally entangled states are pure. The irregularities in the figure are due to the approximative nature of the numerical method used [19].

$$
\rho_{q}=q|W\rangle\langle W|+(1-q)| G H Z\rangle\langle G H Z|,
$$

BSA was calculated in Ref. [4], using the numerical method presented in Ref. [19], and shown to be composed only of maximally entangled states, with respect to either one or two entanglement. Note, nonetheless, that despite $B_{\text {app }}(\rho)$ being an entanglement monotone [2], it is a quite odd quantity, as every entangled pure state is maximally entangled.

An important measure of multipartite entanglement is the generalized robustness of entanglement [20],

$$
R^{k}(\rho)=\min s, \quad \text { s.t. } \frac{1}{1+s} \rho+\frac{s}{1+s} \pi=\sigma,
$$

where $\sigma \in S_{k}(H)$ and $\pi \in D(H)$. It gives good bounds for the maximum fidelity of teleportation, the distillable entanglement, and the entanglement of formation [4], and has important applications in the study of threshold of errors in quantum gates [21]. In Ref. [4], it was shown that $R$ can be written as Eq. (4), with $\mathcal{C}=\{W \mid W \leqslant d I\}$, where $d$ is the dimension of the state space.

Lemma 1. For every state $\rho \in D(H)$,

$$
\max _{\sigma \in S_{k}(H)} \operatorname{Tr}(\rho \sigma) \geqslant \frac{\operatorname{Tr}\left(\rho^{2}\right)}{1+R^{k}(\rho)} .
$$

Proof. From the theory of convex optimization and Lagrange duality [22], the optimal value of the left-hand side (LHS) of Eq. (10) is given by the solution of the following convex problem:

$$
\min \lambda \text { s.t. } \lambda I-\rho \in \mathcal{W}_{k} \text {. }
$$

Let $W=\lambda_{\text {opt }} I-\rho$ be an optimal solution of (11). Since, $W / \lambda_{\text {opt }} \leqslant I$, we find that $R^{k}(\rho) \geqslant \operatorname{Tr}\left(\rho^{2}\right) / \lambda-1$, from which the result follows.

Theorem 5. There do not exist, for any $k$, mixed maximally $k$-entangled states according to the generalized robustness of entanglement. 
Proof. We will prove by contradiction that an optimal entanglement witness of the form (6) does not exist. Assume that $\rho^{\prime}$ is a mixed maximally entangled state with spectral decomposition $\rho^{\prime}=\sum_{i=1}^{m} \lambda_{i}|i\rangle\langle i|$. Then, by the dual definition of $R$, it is easily seen that $\rho=\lambda|1\rangle\langle 1|+(1-\lambda)| 2\rangle\langle 2|$ is also maximally entangled for every $\lambda \in[0,1]$. Thus we consider, without loss of generality, states of rank two. For the generalized robustness, the matrix $D$ of Eq. (6) must satisfy $D \leqslant I$. Hence, since for every entanglement witness $W=(-E) I \oplus D$, with $D \leqslant I, W^{\prime}=(-E) I \oplus I$ is another witness as optimal as $W$ for $\rho$, we may assume throughout this canonical form. Letting $P$ represent the projector onto the support of $\rho, W=I-(1+E) P$. By assumption, $R(P)=2 E$. From lemma 1 , we find that for some $\sigma \in S_{k}(H)$,

$$
\operatorname{Tr}(P \sigma) \geqslant \frac{\operatorname{Tr}\left(P^{2}\right)}{1+R(P)}=\frac{2}{1+2 E} .
$$

Therefore,

$$
\operatorname{Tr}(W \sigma)=1-(1+E) \operatorname{Tr}(\sigma P) \leqslant 1-\frac{2(1+E)}{1+2 E}<0,
$$

which contradicts the fact that $W$ is a $k$-entanglement witness.

In Ref. [4], a family $E_{m: n}$ of infinite entanglement monotones which interpolates between the best separable approximation measure and the generalized robustness was proposed. For fixed $m$ and $n, E_{m: n}$ is given by Eq. (4), with
$\mathcal{C}=\{W \mid-m I \leqslant W \leqslant n I\}$. They provide a tool to the observation of the (smooth) transition between the regime where there exist mixed maximally entangled states and the regime where all MMES are pure. Figure 1 shows the transition for the family of states given by Eq. (8).

In conclusion, we have analyzed the existence of maximally mixed entangled states in the bipartite and multipartite scenarios. In the first scenario, we showed that, although monotonicity under LOCC does not exclude MMES, partial additivity and asymptotic continuity together with monotonicity do. We then extended this result to multipartite systems by showing that maximally entangled (multiparticle) states are pure. It is now time to ask: What are the physical consequences of this result? One can easily note that every pure state must be completely uncorrelated with any other system (if not it should be written as a nontrivial convex combination, which characterizes mixed states). This notion gives us a solid background to propose the faithful polygamy of entanglement, which states that all maximally entangled states are (classically and quantically) uncorrelated with any other system. One can even propose this condition as another requisite for a good multipartite entanglement quantifier. Furthermore, it is also important to stress that this polygamy holds for all kinds of entanglement, that is, every time the system reaches a maximum amount of entanglement according to any partition, it becomes "free" of its environment.

D.C. and F.G.S.L.B. acknowledge financial support from CNPq. The authors thank M.F. Santos for useful discussions.
[1] V. Coffman, J. Kundu, and W. K. Wootters, Phys. Rev. A 61, 052306 (2000); T. J. Osborne and F. Verstraete, e-print quantph/0502176; M. Koashi and A. Winter, Phys. Rev. A 69, 022309 (2004).

[2] S. Karnas and M. Lewenstein, J. Phys. A 34, 318 (2001).

[3] V. Vedral and M. B. Plenio, Phys. Rev. A 57, 1619 (1998).

[4] F. G. S. L. Brandão, e-print quant-ph/0503152, Phys. Rev. A (to be published); F. G. S. L. Brandão and R. O. Vianna, e-print quant-ph/0405096.

[5] G. Adesso and F. Illuminati, e-print quant-ph/0410050.

[6] F. Verstraete, K. Audenaert, and B. De Moor, Phys. Rev. A 64, 012316 (2001); T. C. Wei, K. Nemoto, P. M. Goldbart, P. G. Kwiat, W. J. Munro, and F. Verstraete, ibid. 67, 022110 (2003).

[7] M. A. Nielsen and I. L. Chuang, Quantum Computation and Quantum Information (Cambridge University Press, Cambridge, UK, 2000).

[8] G. Vidal, J. Mod. Opt. 47, 355 (2000).

[9] K. R. Parthasarathy, e-print quant-ph/0405077.

[10] C. H. Bennett, D. P. DiVincenzo, J. A. Smolin, and W. K. Wootters, Phys. Rev. A 54, 3824 (1996).

[11] G. Vidal and R. F. Werner, Phys. Rev. A 65, 032314 (2002).

[12] W. K. Wootters, Phys. Rev. Lett. 80, 2245 (1998); P. Rungta and C. M. Caves, e-print quant-ph/0208002.
[13] M. Horodecki, P. Horodecki, and R. Horodecki, Phys. Rev. Lett. 84, 2014 (2000); M. J. Donald, M. Horodecki, and O. Rudolph, J. Math. Phys. 43, 4252 (2002).

[14] W. Dür and J. I. Cirac, Phys. Rev. A 61, 042314 (2000).

[15] W. Dür, G. Vidal, and J. I. Cirac, Phys. Rev. A 62, 062314 (2000); F. Verstraete, J. Dehaene, B. De Moor, and H. Verschelde, ibid. 65, 052112 (2002).

[16] M. Horodecki, P. Horodecki, and R. Horodecki, Phys. Lett. A 223, 1 (1996).

[17] J. Eisert, K. Audenaert, and M. B. Plenio, J. Phys. A 36, 5605 (2003); M. Horodecki, A. Sen De, and U. Sen, Phys. Rev. A 70, 052326 (2004).

[18] M. Popp, F. Verstraete, M. A. Martin-Delgado, and J. I. Cirac, Phys. Rev. A 71, 042306 (2005); J. Eisert and H.-J. Briegel, ibid. 64, 022306 (2001); T. C. Wei and P. M. Goldbart, ibid. 68, 042307 (2003).

[19] F. G. S. L. Brandão and R. O. Vianna, Phys. Rev. Lett. 93, 220503 (2004).

[20] M. Steiner, Phys. Rev. A 67, 054305 (2003); G. Vidal and R. Tarrach, ibid. 59, 141 (1999).

[21] A. W. Harrow and M. A. Nielsen, Phys. Rev. A 68, 012308 (2003).

[22] S. Boyd and L. Vandenberghe, Convex Optimization (Cambridge University Press, Cambridge, UK, 2000). 